Recepción: 09 / 08 / 2018

Aceptación: 17 / 09 / 2018

Publicación: $01 / 11 / 2018$

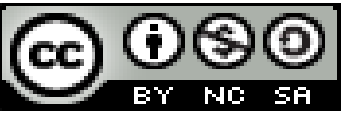

Ciencias económicas y empresariales

Artículo de investigación

\title{
Metodología para la auditoría integral en la efectividad de la gestión de riesgos
}

\section{Methodology for comprehensive audit in risk management}

Metodologia para auditoria abrangente em gerenciamento de riscos

\author{
Gema Viviana Paula-Alarcón ${ }^{\mathrm{I}}$ \\ gemapaula@unach.edu.ec
}

Doris Nataly Gallegos-Santillán II

dgallegos@unach.edu.ec

Correspondencia: gemapaula@unach.edu.ec

I Magíster en Auditoría Integral, Docente Ocasional de la Universidad Nacional de Chimborazo, Riobamba, Ecuador.

II Magíster en Gerencia Empresarial, Doctorado en Gestión de Empresas, Docente de la Universidad Nacional de Chimborazo, Riobamba, Ecuador. 


\title{
Resumen
}

La finalidad del presente trabajo de investigación fue diseñar las bases teóricas para aplicar en la práctica la auditoría integral, que permita la efectividad en la gestión de riesgos, para lo cual se consideró la revisión de estándares internacionales y nacionales en la aplicación de la auditoría integral como un examen que proporciona una evaluación objetiva y constructiva acerca del grado en que los recursos humanos, financieros y materiales son manejados con debidas economías, eficacia y eficiencia, en base a las diferentes modalidades de la auditoría integral como son: auditoría financiera, auditoría de cumplimiento, auditoría de gestión y control interno, tomando en consideración la planeación y la ejecución. El estudio determinó la importancia de los procesos de enseñanza y aprendizaje en cuanto al uso de metodologías de valoración y administración de riesgos empresariales con resultados eficientes y eficaces que garantizan la integralidad de las diferentes auditorias que forman parte de la auditoría integral.

Palabras clave: Auditoría integral; auditoría financiera; auditoría de cumplimiento; auditoría de gestión; control interno; gestión de riesgos.

\begin{abstract}
The purpose of this research work was to design the theoretical basis to apply in practice the comprehensive audit, which allows the effectiveness in risk management, for which the review of international and national standards in the application of the comprehensive audit was considered. as an examination that provides an objective and constructive evaluation of the degree to which human, financial and material resources are managed with due economy, effectiveness and efficiency, based on the different modalities of the comprehensive audit, such as: financial audit, audit of compliance, management audit and internal control, taking into consideration the planning and execution. The study determined the importance of the teaching and learning processes regarding the use of business risk assessment and management methodologies with efficient and effective results that guarantee the integrality of the different audits that are part of the integral audit.
\end{abstract}

Key words: Comprehensive audit; financial audit; compliance audit; management audit; internal control; risk management. 


\section{Resumo}

O objetivo deste trabalho de pesquisa foi projetar a base teórica para aplicar na prática a auditoria abrangente, que permite a eficácia na gestão de riscos, para a qual foi considerada a revisão das normas internacionais e nacionais na aplicação da auditoria abrangente. como um exame que fornece uma avaliação objetiva e construtiva na medida em que os recursos humanos e materiais, financeiros, são tratados com a devida economia, eficácia e eficiência, com base nas diferentes modalidades de auditoria abrangente como: auditoria financeira, Auditoria de auditoria de gestão e controle interno, levando em consideração planejamento e gerenciamento. $\mathrm{O}$ estudo determinou a importância dos processos de ensino e aprendizagem no uso de metodologias de avaliação e gestão de riscos de negócios, com resultados eficientes e efetivos, que garantiram a integralidade das diferentes auditorias que fazem parte da auditoria integral.

Palavras-chave: Auditoria abrangente; auditoria financeira; auditoria de conformidade; auditoria de gestão; controle interno; gestão de riscos

\section{Introducción}

Las nuevas tecnologías empresariales y las corrientes de la reingeniería y calidad total, han motivado a que la Auditoría incursione en nuevos enfoques. La necesidad de disponer de informes integrales de evaluación, se presenta como una alternativa válida para enfrentar los retos del control externo a la organización. Las orientaciones específicas de las auditorías financieras y de gestión, si bien han proporcionado aportes importantes para el mejoramiento de la gestión empresarial pero no cubren todos los enfoques.

Es en este ámbito donde juega un papel fundamental la Auditoría Integral, la cual es utilizada como una herramienta que enfrenta las innovaciones gerenciales y administrativas garantizando la detección de desviaciones o deficiencias de la gestión administrativa, verificando que las acciones se realicen con criterios de eficiencia en el uso de los recursos y logros de objetivos, así como la eficacia en los resultados, la economía en términos de proporcionalidad y su relación costo-beneficio, para promover así la productividad y contribuir al logro de los objetivos.

La auditoría integral constituye una oportunidad a los esquemas de control vigentes en el Ecuador. Su adopción en otros países, demuestra la utilidad de un enfoque completo y holístico 
de la auditoría. Es así, en el presente estudio se presenta una metodología para la auditoría integral en la efectividad de la gestión de riesgos, a través de la definición y objetivos de la auditoría integral, criterios aplicables, metodología e informe. Esta aplicación se realizó en la empresa ENTER SYSTEMS Latinoamericana de Computadoras \& Sistemas Cía. Ltda., ubicada en la ciudad de Riobamba - Ecuador, bajo un enfoque global de análisis, evaluación y mejoramiento de la gestión empresarial privada y organizacional en el sector público. Disponer de una herramienta de soporte gerencial y operativo que cubra todos los frentes de la administración constituye la aspiración técnica de la presente investigación y responde a una inquietud cada vez más evidente de transformar las acciones de control en verdaderos instrumentos de apoyo.

De tal forma que, la metodología aplicada en la auditoría integral, es una herramienta de revisión exhaustiva en la que se analiza la efectividad de los controles gestión de riesgo.

\section{Marco Teórico}

1.1. Descripción conceptual y los criterios principales de todas las auditorías que conforman la auditoría integral

\subsubsection{Auditoría integral.}

Auditoría integral es el examen crítico, sistemático y detallado de los sistemas de información financiero, de gestión y legal de una organización, realizado con independencia y utilizando técnicas específicas, con el propósito de emitir un informe profesional sobre la razonabilidad de la información financiera, la eficacia, eficiencia y economicidad en el manejo de los recursos y el apego de las operaciones económicas a las normas contables, administrativas y legales que le son aplicables, para la toma de decisiones que permitan la mejora de la productividad de la misma.

Blanco, Y (2012) manifiesta que es el proceso de obtener y evaluar objetivamente, en un período determinado, evidencia relativa a la siguiente temática: la información financiera, la estructura de control interno financiero, el cumplimiento de las leyes y regulaciones pertinentes y la conducción ordenada en el logro de las metas y objetivos propuestos; con el propósito de 
informar sobre el grado de correspondencia entre estos temas y los criterios o indicadores establecidos para su evaluación.

Franklin, E (2007) indica que es el examen y evaluación de la planeación, organización, control interno operativo, control interno contable y mejoramiento administrativo, así como el cumplimiento de las disposiciones legales y administrativas aplicables a un área o programa.

\subsubsection{Auditoría Financiera}

Sánchez, G (2006) manifiesta que en un enfoque universal, la auditoría es el examen integral sobre la estructura, las transacciones y el desempeño de una entidad económica, para contribuir a la oportuna prevención de riesgos, la productividad en la utilización de los recursos y el acatamiento permanente de los mecanismos de control implantados por la administración. Esta definición, cosechada a lo largo de la práctica profesional, involucra también a los auditores externos que dictaminan estados financieros, y refleja una parte importante de las expectativas del mercado. Hay que asumirlo: el dictamen por sí solo no basta para que los clientes estén satisfechos.

A su vez, la auditoría de estados financieros puede definirse como el examen de los estados financieros básicos preparados por la administración de una entidad económica, con objeto de opinar respecto a si la información que incluyen está estructurada de acuerdo con las normas de información financiera aplicables a las características de sus transacciones.

El trabajo profesional que conduce al dictamen es largo, interesante y pletórico de retos; debe llevarse a cabo en varias etapas que comienzan en los albores del ejercicio sujeto a examen, y concluye durante los primeros meses del siguiente. La aplicación de las normas de auditoría comienza antes de que el cliente contrate al auditor. A continuación veremos por qué y cuáles son las etapas del proceso.

\subsubsection{Auditoría de Cumplimiento}

Blanco, Y (2012) manifiesta que la Auditoria de Cumplimiento es la comprobación o examen de las operaciones financieras, administrativas, económicas y de otra índole de una entidad para establecer que dichas operaciones se han realizado conforme a las normas legales, reglamentarias, estatutarias y de procedimientos que le son aplicables. Esta auditoría se practica 

mediante la revisión de los documentos que soportan legal, técnica, financiera y contablemente las operaciones para determinar si los procedimientos utilizados y las medidas de control interno están de acuerdo con las normas que le son aplicables y si dichos procedimientos están operando de manera efectiva y son adecuados para el logro de los objetivos de la entidad.

Whittington, R y Kurt, P (1999) manifiesta que la auditoría de cumplimiento comprende la prueba y la presentación de informes sobre si una organización ha cumplido los requisitos de las diversas leyes, regulaciones y acuerdos.

\subsubsection{Auditoría de Gestión}

Velásquez, M (2012) manifiesta que la auditoría de gestión es un proceso que nos permite examinar y evaluar las actividades realizadas en una organización, sector, programa, proyecto $\mathrm{u}$ operación. Actividad que sobre la base de un modelo y un marco normativo, permite determinar el grado de eficiencia, eficacia, y calidad en la utilización de los recursos disponibles, el desarrollo de procesos y la entrega de productos o de servicios, acordes con las necesidades de los clientes, los mismos que podrán ser mejorados en forma continua, a través de la aplicación de las recomendaciones emanadas del informe de auditoría.

Todo proceso de auditoría se basa en criterios o estándares que permitan medir la calidad del trabajo efectuado en cada una de las fases, las mismas que constituyen una cadena de valor, que se inicia con el diagnóstico preliminar en el cual se tiene un primer acercamiento a la empresa o institución, se podría decir que el auditor empieza a medir potencialmente el nivel de riesgo inherente.

En conclusión la Auditoría de Gestión es la evaluación o examen sistemático de la información administrativa, operativa y financiera presentada por los administradores de una organización, realizada con posterioridad a su ejecución, para obtener evidencia suficiente del grado de eficiencia, eficacia y calidad en el logro de metas y objetivos propuestos.

\subsubsection{Control Interno}

Mantilla, S (2011) manifiesta que el control interno significa cosas distintas para diferente gente. Ello origina confusión entre personas de negocios, legisladores, reguladores y otros. Dando como resultado malas comunicaciones y distintas expectativas, lo cual origina problemas. Tales 
problemas se entremezclan cuando el término, si no es definido claramente, se escribe en leyes, regulaciones o reglas.

\subsubsection{Gestión de riesgos en la auditoría}

En la literatura científica existe una amplia valoración sobre los riesgos y su gestión, pero con un marcado enfoque de la administración de riesgos financieros y los asociados al Sistema de Control Interno, no siendo tan variada y actualizada la relativa a los riesgos de auditoría.

La Administración de Riesgos, según Alonso, A (2007), es una actividad consustancial a la misma naturaleza humana y, enfocada desde esta óptica, una práctica tradicional que se puede encontrar en cualquier lugar y momento histórico. En el mundo, se ha practicado desde hace mucho tiempo. Sin embargo, con la aplicación de las técnicas modernas de la teoría de las probabilidades, del seguro y otros instrumentos afines, este trabajo ha tomado un cuerpo debidamente formalizado, constituyendo un instrumento indispensable en toda evaluación sería sobre el desenvolvimiento esperado de cualquier proyecto productivo.

2. Metodología de la auditoría integral en la efectividad de la gestión de riesgos

\subsection{Fases de la Auditoría}

Para tener una adecuada metodología, es importante tomar en cuenta que la planeación y ejecución de la auditoría integral, se puede agrupar en cinco fases diferentes. Una representación esquemática de estas fases es la siguiente:

\section{Cuadro 1}

\section{Fases de la Auditoría}

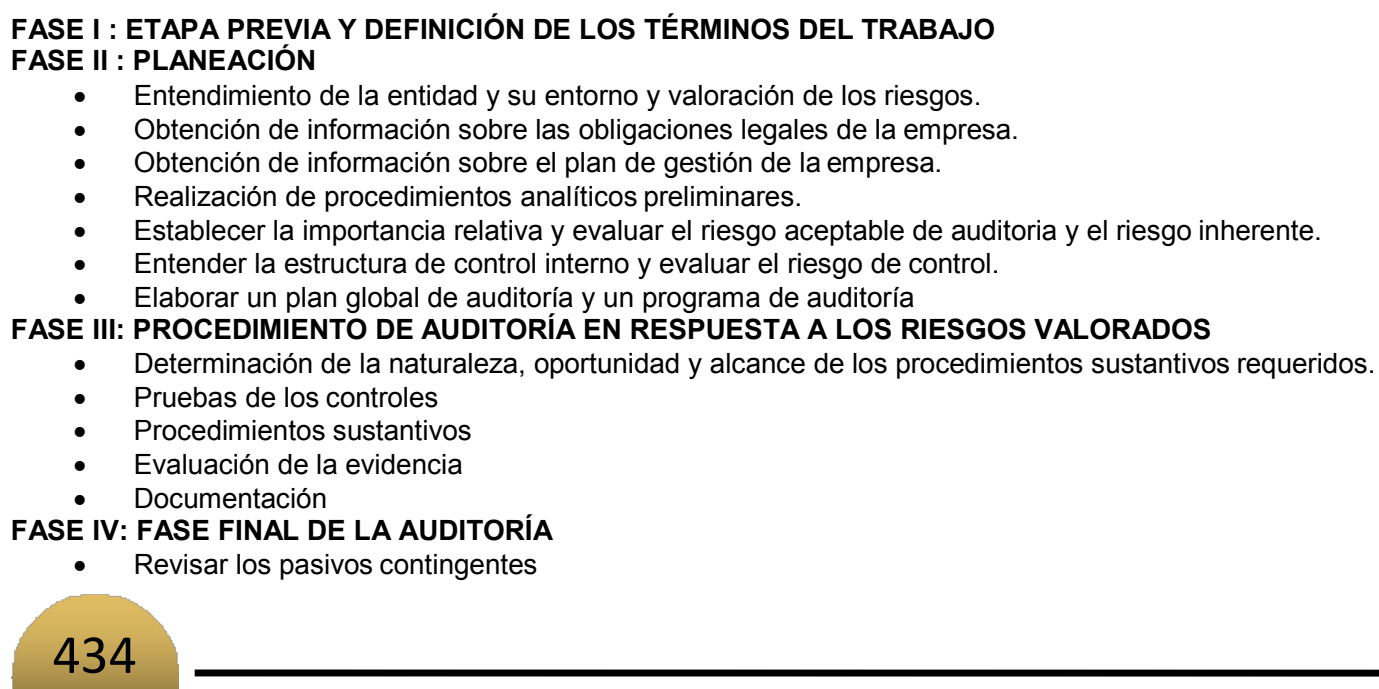

- Entendimiento de la entidad y su entorno y valoración de los riesgos.

- Obtención de información sobre las obligaciones legales de la empresa.

- Obtención de información sobre el plan de gestión de la empresa.

- Realización de procedimientos analíticos preliminares.

- Establecer la importancia relativa y evaluar el riesgo aceptable de auditoria y el riesgo inherente.

- Entender la estructura de control interno y evaluar el riesgo de control.

- Elaborar un plan global de auditoría y un programa de auditoría

FASE III: PROCEDIMIENTO DE AUDITORÍA EN RESPUESTA A LOS RIESGOS VALORADOS

- Determinación de la naturaleza, oportunidad y alcance de los procedimientos sustantivos requeridos.

- Pruebas de los controles

- Procedimientos sustantivos

- Evaluación de la evidencia

- Documentación

FASE IV: FASE FINAL DE LA AUDITORÍA

- Revisar los pasivos contingentes 
- Revisar los acontecimientos posteriores

- Acumular evidencias finales:

- Evaluar el supuesto de empresa en marcha.

- Obtener carta de presentación o certificaciones de los estados financieros.

- Revisar los estados financieros y verificar que las cifras finales concuerdan con ellos.

- Construir los indicadores esenciales para evaluar el desempeño de la administración en el cumplimiento de los objetivos y metas

- Emitir el informe de auditoría integral

- Discusión del informe con la Administración o Comité de Auditoría

FASE V: CONTROL DE CALIDAD DE LOS SERVICIOS

Fuente: Yanel Blanco Luna (2012): Auditoría integral normas y procedimientos, 2da edición

2.2. Matriz de riesgos y enfoque de la auditoría

La teoría, elementos y modelo de la matriz de riesgo y enfoque de la auditoría es una adaptación del contenido en el texto complementario: Auditoría un nuevo enfoque empresarial, Slosse, C. y otros

La "Matriz de Riesgo y Enfoque de la Auditoría" es un producto del proceso de calificación del riesgo efectuada a través de los cuestionarios, controles clave y pruebas de recorrido y la aplicación de la matriz de calificación del nivel de confianza y riesgo, a nivel global y sobre cada componente.

\subsubsection{Finalidad}

La "Matriz de Riesgo y Enfoque de la Auditoría" informa y resume los resultados de la evaluación del control interno en lo que respecta al riesgo inherente y de control existente en los componentes seleccionados.

A la vez que es un producto, este instrumento de Gerencia, sirve de insumo para efectuar el monitoreo y seguimiento de las actividades asignadas a los auditores, en los "programas de auditoría", en tanto deben mantener, durante el trabajo de campo, el enfoque determinado en dicha matriz, sin desviarse del contenido de los programas y evitar la realización de procedimientos diferentes a los asignados.

Además de ayudar en el control de calidad sobre los resultados de la auditoría, sirve como elemento de planificación de futuras acciones de control, ya que le otorga el conocimiento del nivel de riesgo al que el auditor se enfrenta.

\subsubsection{Elementos}


La "Matriz de calificación de riesgos, controles clave y enfoque de la auditoría" contiene los siguientes elementos:

- Nombre del componente

- Saldos (afirmaciones) que constan en los estados financieros

- Calificación del riesgo inherente y riesgo de control del componente.

- Argumentos de dicha calificación

- Los “controles clave", procesos, políticas y normas probadas y que servirán para probar las siguientes muestras

- El enfoque de la auditoría, sea este de "sistemas o cumplimiento" o sustantivo, que se aplicará en la fase de ejecución, mediante los programas de auditoría

\section{Cuadro 2}

Matriz de Calificación de Riesgos 
Metodología para la auditoría integral en la efectividad de la gestión de riesgos

\begin{tabular}{|c|c|c|c|c|c|c|c|}
\hline \multirow{2}{*}{\multicolumn{8}{|c|}{$\begin{array}{c}\text { EMPRESA AUDITADA } \\
\text { AUDITORIA FINANCIERA }\end{array}$}} \\
\hline & & & & & & & \\
\hline \multicolumn{8}{|c|}{$\begin{array}{l}\text { AUDITORIA FINANCIERA } \\
\text { PERIODO: DEL }\end{array}$} \\
\hline \multicolumn{8}{|c|}{ MATRIZ DE CALIFICACION DE RIESGOS, CONTROLES CLAVE Y ENFOQUE DE LA AUDITORIA } \\
\hline COMPONENTE & & & & & & $\begin{array}{c}\text { ENFOQUE } \\
\text { CUMPLIMIENTO }\end{array}$ & $\begin{array}{l}\text { ENFOQUE } \\
\text { SUSTANTIVO }\end{array}$ \\
\hline EFECTIVO & \multicolumn{4}{|c|}{ NIVEL DE RIESGO } & $\begin{array}{l}\text { CONTROL } \\
\text { CLAVE }\end{array}$ & $\begin{array}{c}\text { Verificación } \\
\text { Seguimiento } \\
\text { Comprobación de } \\
\text { controles }\end{array}$ & $\begin{array}{l}\text { Confirmaciónes } \\
\text { Constatación } \\
\text { Inspección } \\
\text { Conciliación }\end{array}$ \\
\hline AFIRMACIONES & \multicolumn{4}{|c|}{ Nivel de Riesgo y Argumentación } & & & \\
\hline $1 \% 410.000$ USD & Inherente & Bajo & Control & Bajo & & & \\
\hline \multirow[b]{2}{*}{ CAJA } & Inherente & Bajo & Control & Bajo & & & \\
\hline & & & & & Arqueo & $\begin{array}{l}\text { Pruebas de } \\
\text { Recorrido: } \\
\text { Política de Manejo } \\
\text { de Fondos }\end{array}$ & \\
\hline \multirow[b]{2}{*}{ FONDOS FIJOS } & Inherente & Bajo & Control & Bajo & & & \\
\hline & \multicolumn{2}{|c|}{$\begin{array}{l}\text { Falta de formalidad en la } \\
\text { asignación de fondos }\end{array}$} & & & Arqueo & $\begin{array}{l}\text { Pruebas de } \\
\text { Recorrido: } \\
\text { Política de Manejo } \\
\text { de Fondos }\end{array}$ & \\
\hline \multirow[b]{2}{*}{ BANCOS } & Inherente & Bajo & Control & Bajo & & & \\
\hline & & & & & Conciliación & $\begin{array}{l}\text { Pruebas de } \\
\text { Recorrido: } \\
\text { Sistema de } \\
\text { Cobranzas }\end{array}$ & \\
\hline
\end{tabular}

Fuente: Slosse, C. y otros (1995), Auditoria, Un nuevo enfoque empresarial, Argentina, Ediciones Macchi

\subsection{Estructura del Informe de Auditoría Integral}

El informe de auditoría integral debe someterse a revisiones que garanticen su objetividad con respecto a los temas evaluados. Además debe comunicarse al auditado desde el inicio del proceso y cuando se hayan determinado hechos relevantes. 
Cuadro 3

\section{Estructura del Informa de Auditoría Integral}

- $\quad$ Tema o asunto determinado.
- $\quad$ Parte responsable
- $\quad$ Responsabilidad del auditor
- $\quad$ Ostándares, normas de desempeño aplicables o criterios.
- Opinión sobre la razonabilidad de los estados financieros.
Otras conclusiones

Fuente: Subía Guerra, Jaime Cristóbal. Informe de Auditoría Integral. Guía Didáctica. Editorial de la Universidad Técnica Particular de Loja.

\subsection{Cadena de Valor}

"Porter define el valor como la suma de los beneficios percibidos que el cliente recibe menos los costos percibidos por él al adquirir y usar un producto o servicio. La cadena de valor es esencialmente una forma de análisis de la actividad empresarial mediante la cual descomponemos una empresa en sus partes constitutivas, buscando identificar fuentes de ventaja competitiva en aquellas actividades generadoras de valor. Esa ventaja competitiva se logra cuando la empresa desarrolla e integra las actividades de su cadena de valor de forma menos costosa y mejor diferenciada que sus rivales. Por consiguiente, la cadena de valor de una empresa está conformada por todas sus actividades generadoras de valor agregado y por los márgenes que éstas aportan.” http://www.3w3search.com/Edu/Merc/Es/GMerc081.htm

Dentro de una empresa existen una variedad de actividades, el conjunto de estas actividades forman los procesos, muchos de ellos son generadores de valor para la empresa. La cadena de valor define en forma clara y resumida las actividades de la empresa, mediante la utilización de gráficos en la que se detallan los distintos procesos que se realizan y que generan valor. Enter Systems Latinoamericana de Computadoras \& Sistemas Cía. Ltda. realiza sus actividades en base a la siguiente cadena de valor. 


\section{Figura 1}

\section{Cadena de Valor}

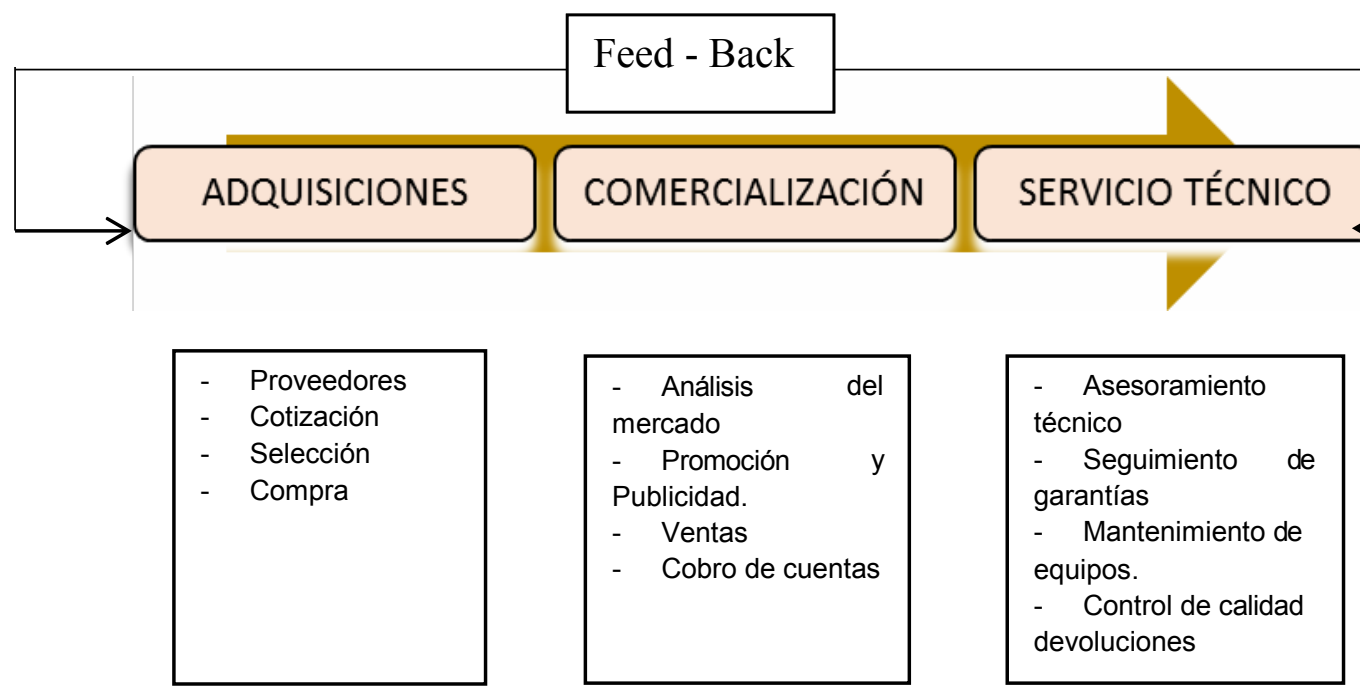

\section{ÁREA ADMINISTRATIVA}

\section{ÁREA FINANCIERA}

Fuente: Elaboración propia de la autora

\subsubsection{Procesos gobernantes}

A la cadena de valor de Enter Systems Latinoamericana de Computadoras \& Sistemas Cía. Ltda., que encierran los procesos básicos y de apoyo, se incluye los procesos gobernantes. Estos procesos son de suma importancia para la toma de decisiones y cuyo resultado afecta a toda la empresa, este proceso incluye actividades a nivel gerencial, y está integrado por los siguientes niveles:

- Junta General de Accionistas

- $\quad$ Presidente

- $\quad$ Gerencia General 
Figura 2

Cadena de Valor procesos gobernantes
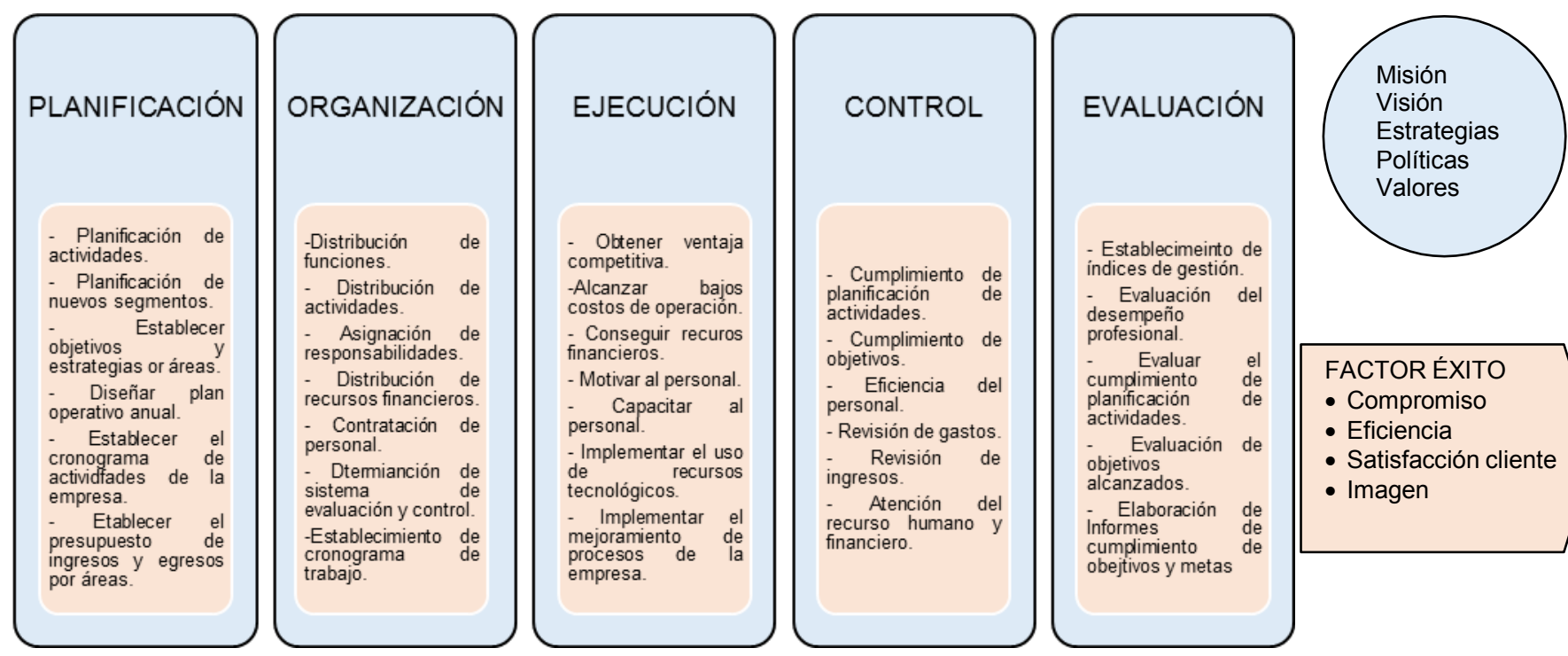

Fuente: Elaboración propia de la autora

\subsubsection{Procesos básicos o agregadores de valor}

Son los procesos operativos que agregan valor, conciernen a las actividades de cada área y orientan su actividad. Los procesos básicos que agregan valor:

- $\quad$ Adquisiciones

- Comercialización

- $\quad$ Servicio Técnico 


\section{Adquisiciones}

\section{Figura 3}

Cadena de Valor procesos básicos - adquisiciones

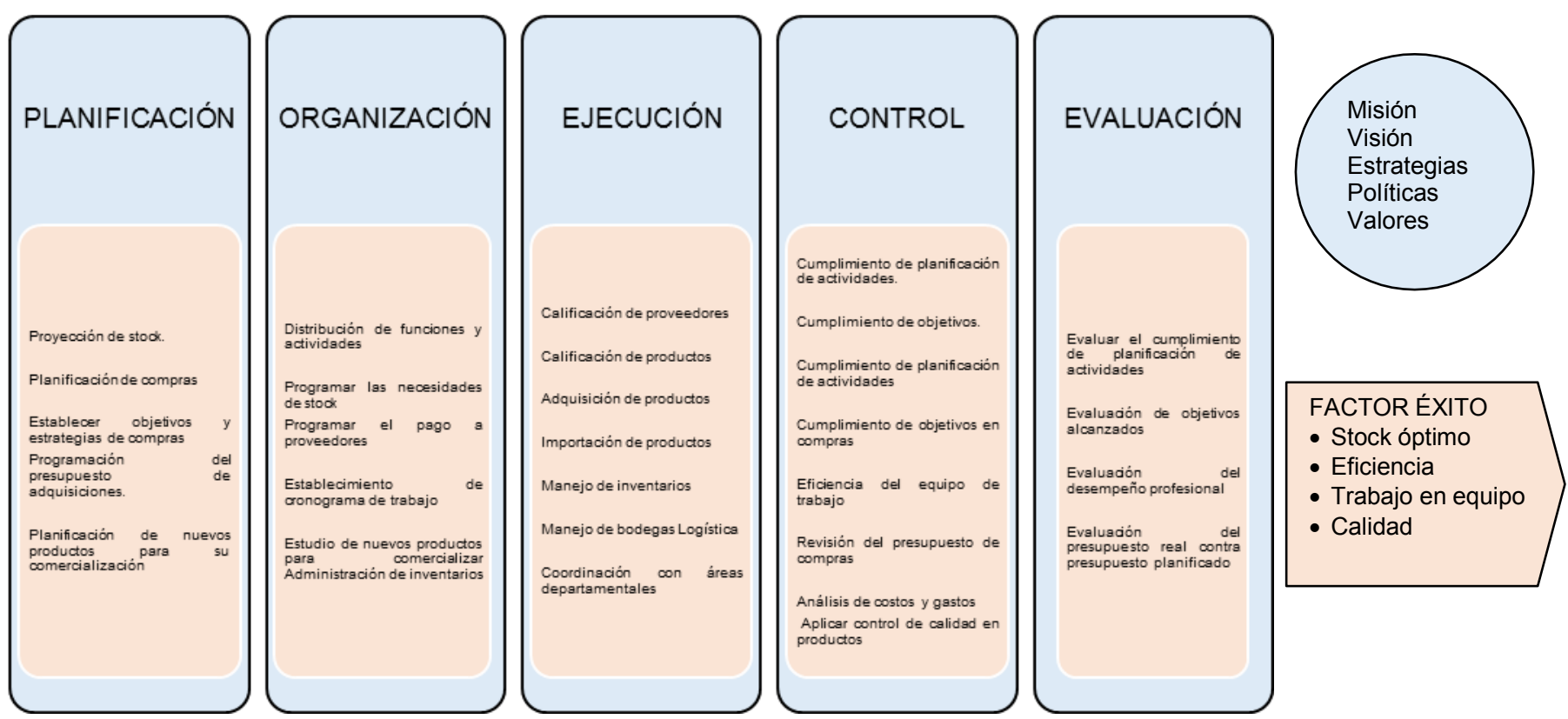

Fuente: Elaboración propia de la autora

\section{Comercialización}

\section{Figura 4}

Cadena de Valor procesos básicos - comercialización
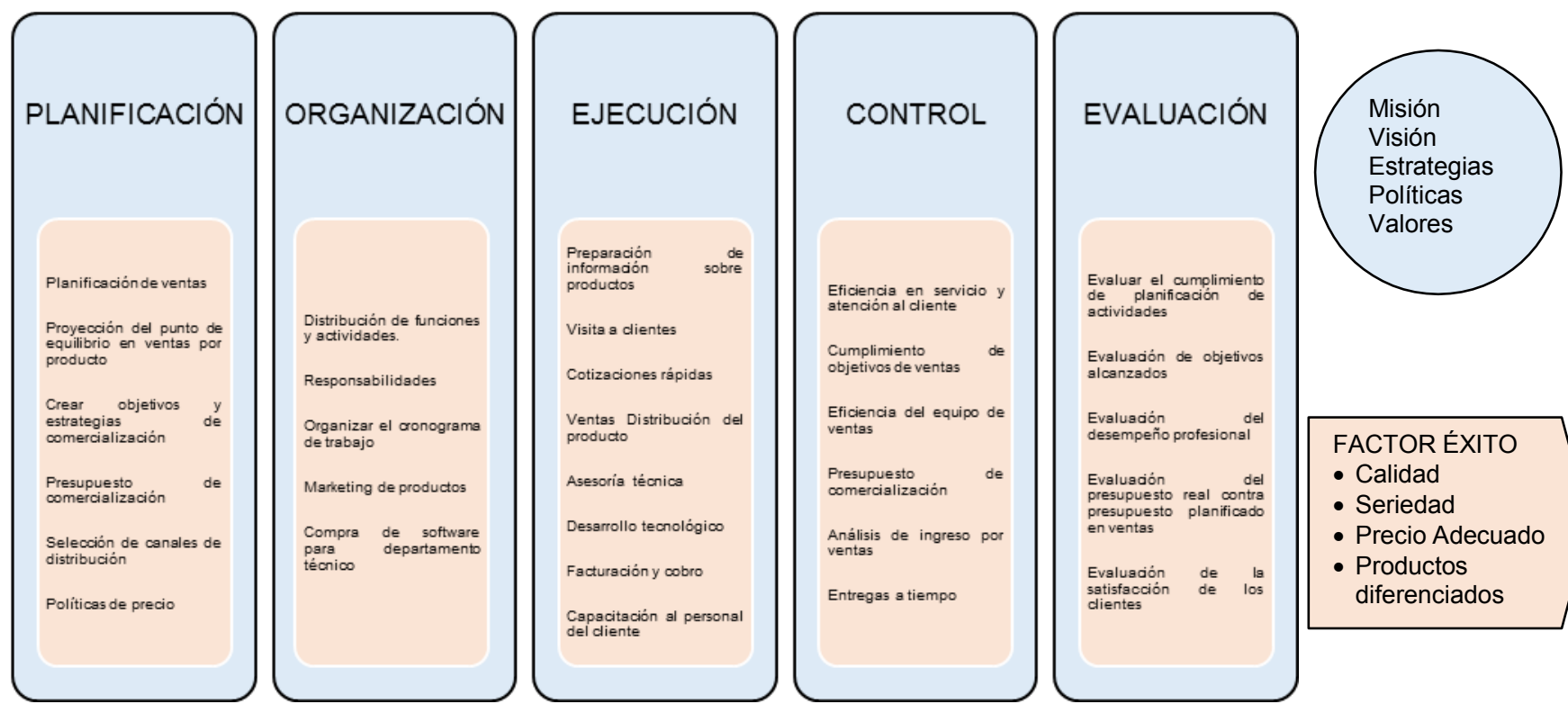

Fuente: Elaboración propia de la autora

FACTOR ÉXITO

- Calidad

- Seriedad

- Precio Adecuado

- Productos diferenciados 


\section{Servicio Técnico}

\section{Figura 5}

Cadena de Valor procesos básicos - servicio técnico

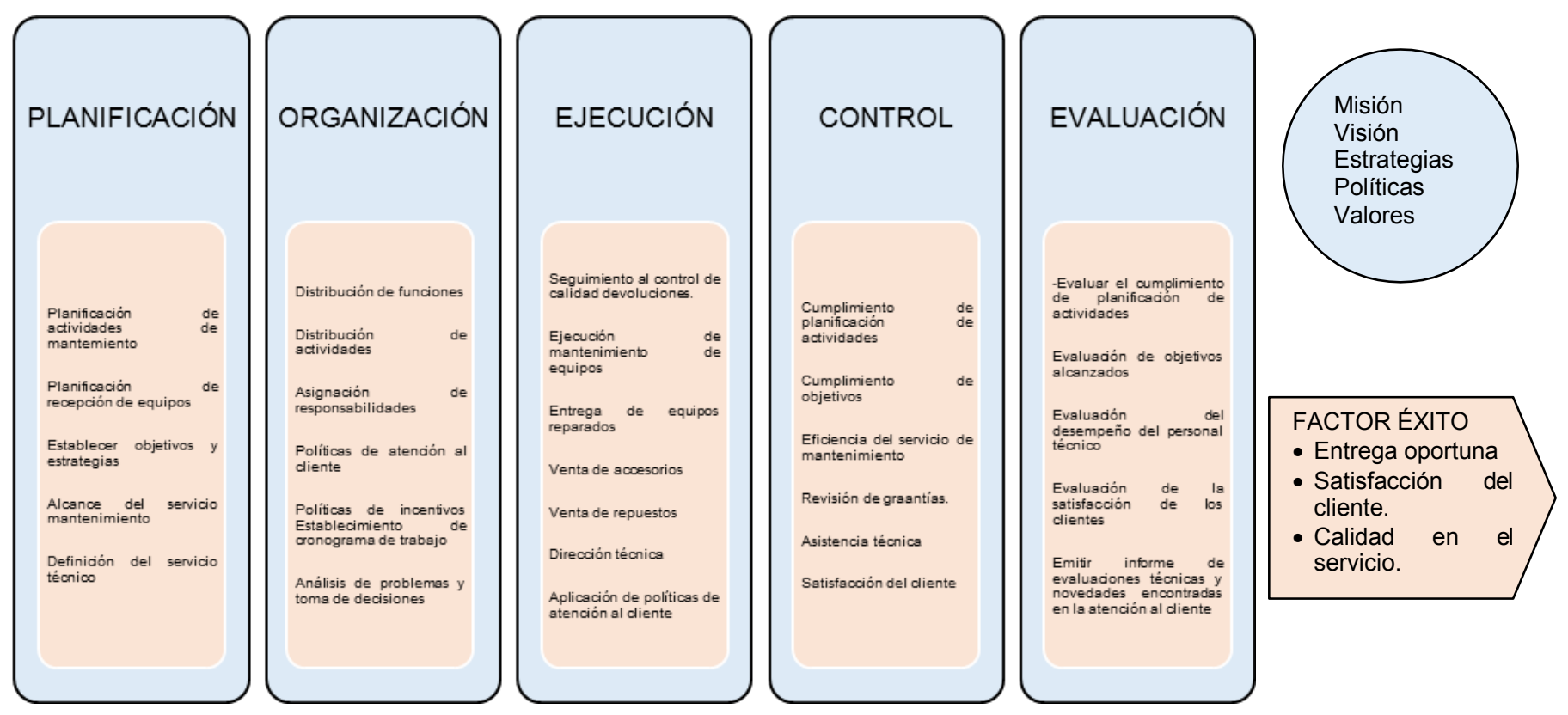

Fuente: Elaboración propia de la autora

\subsubsection{Procesos habilitantes}

Son los procesos que no agregan valor en forma directa, sino que refuerzan proporcionando los recursos necesarios para que los macroprocesos básicos puedan generar valor. Las áreas de habilitantes de la empresa son:

- $\quad$ Área Administrativa

- $\quad$ Área Financiera 


\section{Área Administrativa}

Figura 6

Cadena de Valor procesos habilitantes - área administrativa
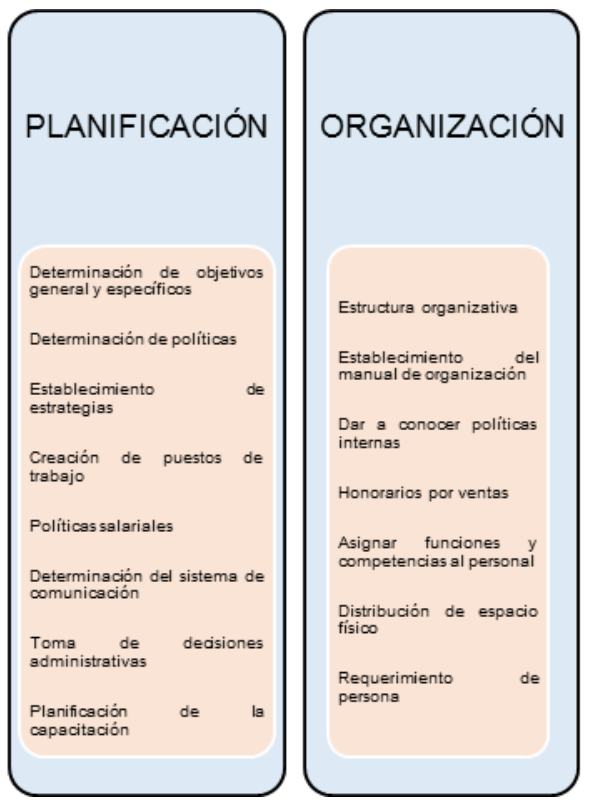
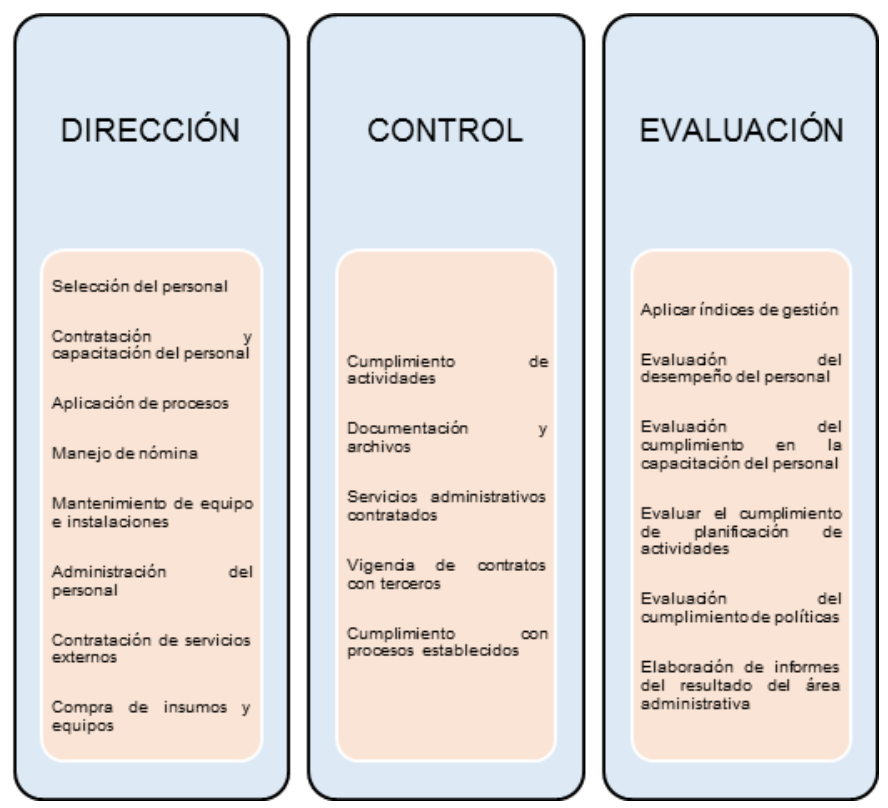

Fuente: Elaboración propia de la autora

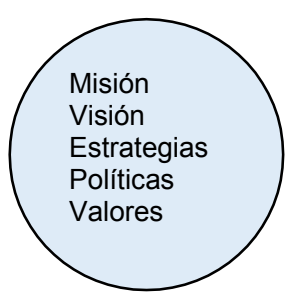

\section{Área Financiera}

Figura 7

Cadena de Valor procesos habilitantes - área financiera
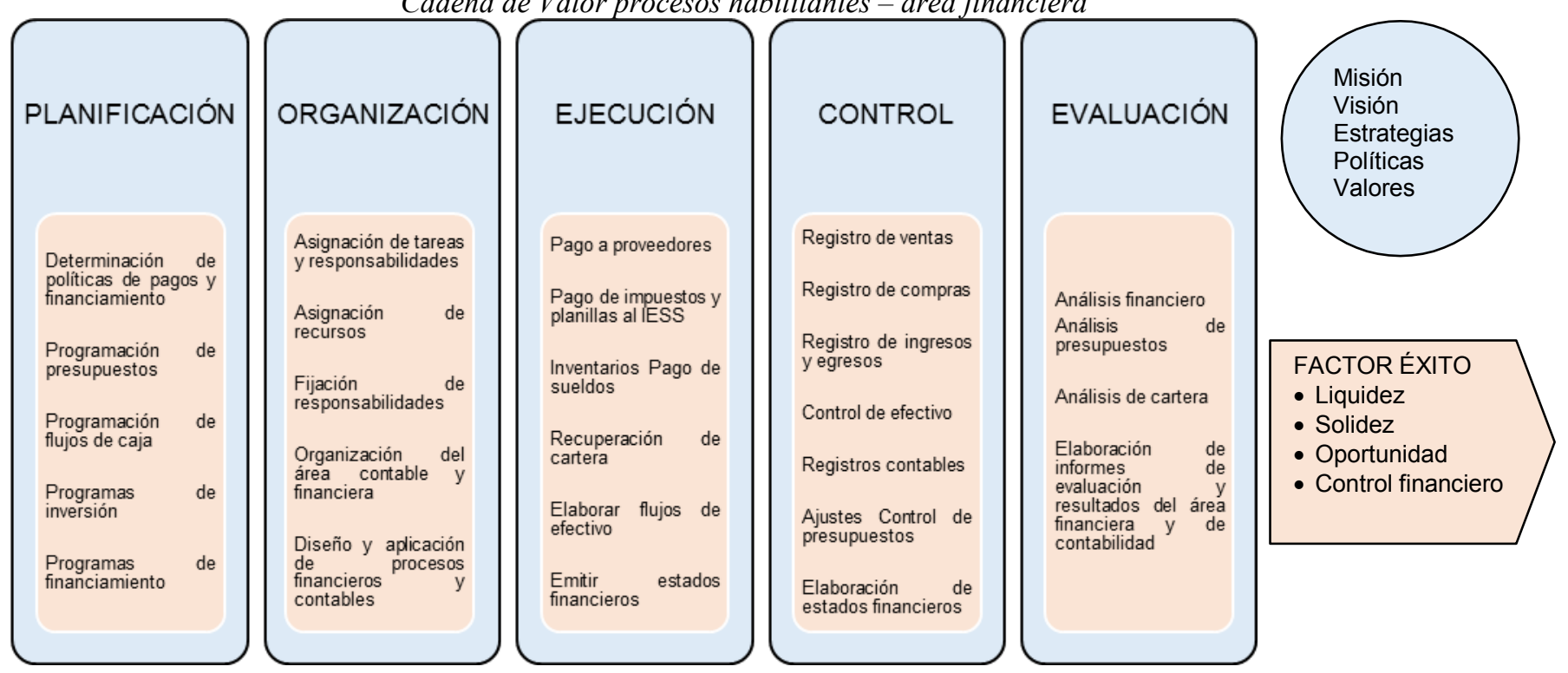

Fuente: Elaboración propia de la autora 
Cabe señalar que hay diferentes metodologías de análisis de riegos y que estas varían de acuerdo a las características de cada entidad, es por ello que es apropiado escoger la metodología idónea para realizar el análisis y su impacto. Entonces es necesario entender que metodologías de análisis de riesgos garanticen que la auditoría integral es una evaluación al sistema de control en funcionamiento revisando hechos que han ocurrido.

El trabajo de investigación permitió determinar la importancia para la enseñanza - aprendizaje metodologías de valoración y administración de riesgos empresariales con resultados eficientes y eficaces que garantizan la integralidad de las diferentes auditorias que forman parte de la auditoría integral.

\section{Conclusiones}

1. La auditoría integral permitirá que ENTER SYSTEMS LATINOAMERICANA DE COMPUTADORAS \& SISTEMS CÍA LTDA., mediante la utilización y aplicación de varias técnicas, pruebas y procedimientos de auditoría integral, pueda lograr la eficiencia en la gestión de riesgos.

2. La auditoría integral permite determinar a través de un examen analítico, sistemático y continuo, áreas críticas o procedimientos deficientes mediante la aplicación de pruebas y técnicas, así como también la aplicación de normas y leyes para emitir un informe el profesional que ayudara a la consecución de los objetivos por ende a la mejor continua de la compañía.

3. El control interno es el conjunto de medidas, políticas y procedimientos establecidos en las empresas, que precisa el desarrollo de las actividades y el establecimiento de relaciones de conducta entre personas y lugares de trabajo en forma eficiente, promover eficiencia en las operaciones.

4. Toda organización, se encuentra sujeta a leyes y regulaciones cuya inobservancia puede poner en peligro la actividad o generar pérdidas por multas y otras sanciones, así como afectar la imagen, credibilidad y prestigio, estos incumplimientos son ocasionados muchas veces por falta de información o desconocimiento de los responsables de cumplir con las regulaciones o no contar con sistemas de supervisión y control que garanticen sus cabal cumplimiento. 
5. En la actualidad al evaluar aspectos y situaciones de carácter financiero, tiene como finalidad, obtener certeza en la orientación de las acciones que les permitan optimizar el uso de sus recursos y obtener resultados confiables de las operaciones que realizan.

6. La auditoría de gestión es un proceso que permite examinar y evaluar las actividades realizadas de una organización, sector, programa, proyecto $\mathrm{y}$ operación, sobre la base de un modelo y un marco normativo.

\section{Referencias Bibliográficas}

[1]. ALVAREZ Francisco, Calidad y Auditoría en Salud, ECOEDICIONES, 2003.

[2]. BELTRÁN c., JESÚS M. (1998): Indicadores de gestión, Herramientas para lograr la competitividad.

[3]. BLANCO LUNA, Yanel (2012): Auditoría integral normas y procedimientos, 2da edición, Colombia, ECOE EDICIONES

[4]. CANO C., Miguel (2004): Auditoría Forense, Colombia, ECOE EDICIONES

[5]. CEPEDA, Gustavo (2002): Auditoría y Control Interno, Mc. Graw Hill, Colombia

[6]. COTECNA, Quality Resources Inc, Curso de Auditor Líder de Sistemas de Gestión de Calidad ISO 9001:2000, Quito-Ecuador 2005.

[7]. ESTUPIÑAN GAITAN, Rodrigo (2007): Pruebas selectivas en la auditoría, Colombia, ECOE EDICIONES

[8]. FRANKLIN, Enrique Benjamín (2007): Auditoría Administrativa, Gestión Estratégica del Cambio, Mèxico, Prentice Hall,

[9]. Harvard Business School Press. The Balanced ScoredCard. (1996): Translating Strategy into Action, Boston.

[10]. INEN, Norma ISO 19011, Directrices para la Auditoría de Sistemas de la Calidad.

[11]. ISO, Normas Internacionales 9000, 9001, 9004 y 19011. 
[12]. KAPLAN, Robert y NORTON, David. (2001): El Cuadro de Mando Integral Balanced Scorecard, Boston, USA: Harvard Business Publishing Corporation.

[13]. KAPLAN, Robert y NORTON, David P. (2001): Cómo utilizar el cuadro de mando integral, España, Editorial Gestión 2000

[14]. MANTILLA, Samuel Alberto (2005): Auditoría del Control Interno, Colombia, ECOE EDICIONES

[15]. MILLS, David (2000, 2003): Manual de Auditoría de la Calidad. España, Editorial Gestión 2000,

[16]. MALDARRIAGA, Juan, (2004): Manual Práctico de Auditoría, DEUSTO, España

[17]. SÀNCHEZ CURIEL, Gabriel (2006): Auditoría de estados financieros, Prentice Hall, México

[18]. SENLLE, Andrés (2000, 2003): Evaluar la Gestión y la Calidad, Editorial Gestión.

[19]. SUBÍA, Guerra, Jaime (2007): Maestría en Auditoría de Gestión de la Calidad, Informe y proceso sistemático de la auditoría, Universidad Técnica Particular de Loja, Loja- Ecuador.

[20]. TRISCHLER, William (2003): Mejora del valor añadido en los procesos, España, Editorial Gestión 2000

[21]. TRISCHLER, William (2000, 2003): Mejora de Valor Añadido en los Procesos, Editorial Gestión.

[22]. WADWWORTH, Harrison, STEPHENS y GODFREY, (2005): Métodos de control de calidad, Compañía Editorial Continental. México 